\title{
The Teaching Method Reformation for the Artificial Intelligence Course
}

\author{
Ling $\mathrm{Lu}^{1, \mathrm{a}}$, Yin Gui ${ }^{1, \mathrm{~b}}$, LiHua $\mathrm{Li}^{1, \mathrm{c}}$ \\ 1 School of Information Engineering, East China Institute of Technology \\ NanChang, JiangXi, 330013, China

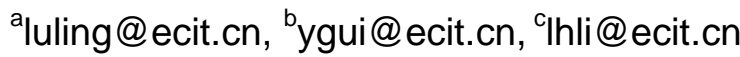

Keywords: artificial intelligence; application example; program design; teaching training system

\begin{abstract}
According to the curriculum and the characteristics of artificial intelligence, we used auxiliary teaching system to reform the teaching method of artificial intelligence course. In the process of teaching, we used a large number of application examples to make abstract theory into the visualization and used the demonstration of algorithm to reify the complex problem. The students can feel the charm of the artificial intelligence technology. The training system was used to make students autonomous learning. Through many years of application, our method can improve the students' interest and ability in learning.
\end{abstract}

\section{Introduction}

In recent decades, the artificial intelligence technology is applied widely, a lot of engineering universities set artificial intelligence course and as one of major courses of information specialty. But the artificial intelligence of teaching exists difficult to learn and teach problem. The main reason is that the course of highly integrated, complicated contents, students are required to have a basic knowledge, learning bring great challenges. If the teaching method is improper, the students will feel very abstract and gradually lose confidence in the course of the study. In order to solve the question, many teachers used of various methods of teaching reform. Yang[1] considered in teaching objectives, course content, selection of subject matter, teaching method and examination form and so on. Trying to work hard for developing new teaching thought, form distinct teaching style and obtain well teaching effect. Liu[2] presented a kind of hierarchical teaching mode and discussed the experimental design of artificial intelligence foundation. Xie[3] presented the construction of artificial intelligence course teaching case base and the case teaching practice plan. $\mathrm{Ma}[4]$ combined with the practice of the teaching, textbooks chosen, teaching arrangement, teaching means and discussed the teaching methods of artificial intelligence. According to the characteristics of the course and teaching content, Wang[5] used case teaching method, heuristic teaching method and project teaching method. This method can effectively mobilize the enthusiasm and initiative of students, deepen the students' understanding and use of knowledge. In practical, Duan[6] pointed out that centralized experimental class cannot give full play to the students' personality and to facilitate students to exchange knowledge, double main body consciousness and the silent teaching method combining teaching method is put forward, to carry out the same experimental subject casually experimental teaching mode and the topic of open experimental teaching measures extension, from the many aspects to improve the students' experiment teaching quality and students to solve the problem. Chen[7] used of the problem-based teaching method in the course teaching of artificial intelligence and to cultivate the students' autonomous learning ability, creation ability and practice ability, so as to achieve the goal of the educational reform of the subject.

We synthesize a variety of methods and use of the auxiliary teaching system that is designed by us. Our method can stimulate students' interest in learning. It can improve students' learning ability, practical ability and innovation ability. 


\section{Artificial intelligence programming}

The choice of programming language. At present in colleges and universities of our country in artificial intelligence course in common software processing platform basically there are two kinds: Prolog and $\mathrm{C}++$.

Prolog is a logic programming language. It is built on the basis of the theory of logic, which was originally applied to the research field of natural language. Now it is used in the study of artificial intelligence widely. For example it can be used to build the expert system, natural language understanding, intelligent knowledge base, etc.. When student use the programming language, they can quickly complete the artificial intelligence results, but on the artificial intelligence algorithm and no further study and consolidation, the software only to researchers study of artificial intelligence, but not for learning in the computer professional students.

$\mathrm{C}++$ is not a programming language for artificial intelligence. So student must write all algorithms about the artificial intelligence. Although it will affect the development efficiency of the user, but students can consolidate the theoretical knowledge and understanding of the artificial intelligence method. For the computer professional students, it can improve the students' programming skills.

We select Visual C++6.0 because the students have to write code from the base algorithms. So the students can understand the artificial intelligence methods and algorithms. If students just stay in the understanding of the theoretical formula, they will form a general understanding and memory that is easy to forget.

The teaching content about program design. The main principle of the design of artificial intelligence program is that the program code is not long and the teaching content is emphasis. So teacher can explain clearly and students can understand easily in the classroom. The content includes: tree state graph search, genetic algorithm, machine reasoning based on generative, etc.. The teacher should explain the idea of programming and do not introduce the program codes. This goal is to promote students to understand the algorithm and improve the students' ability to program design

Program demonstration content. Our teaching content is in addition to the introduction of the program design methods and ideas for artificial intelligence, but also the use of our algorithm demo teaching software to demo the actual effect of the artificial intelligence algorithm. We combine algorithm with examples and make the abstract theory of specific image. The students can feel the charm of the artificial intelligence technology and their learning interest is stimulated. So the efficiency of classroom teaching is improved greatly.

The principle of the design of the software is that input data are some parameters in algorithm. The students can experience the practical meaning of various parameters. For example, in and-or graph search, nodes and their and-or logical relation may be changed (see Fig 1). In A* algorithm, the start position, end position and obstacle position may be changed (see Fig 2). In Fig.2, "X" indicates the obstacle and " 0 " indicates the route. Through the demonstration system shows the digital mobile process, students can realize the effect of the intelligent algorithm. The program demonstration software content more than the program design teaching content. It covers almost all teaching content. For example, state graph search mainly includes graph search (tree search and line search), the tree state graph search (breadth-first search, depth-first search and best-first search), and-or graph search, minimum cost search, $A^{*}$ algorithm, game tree search, etc.. 


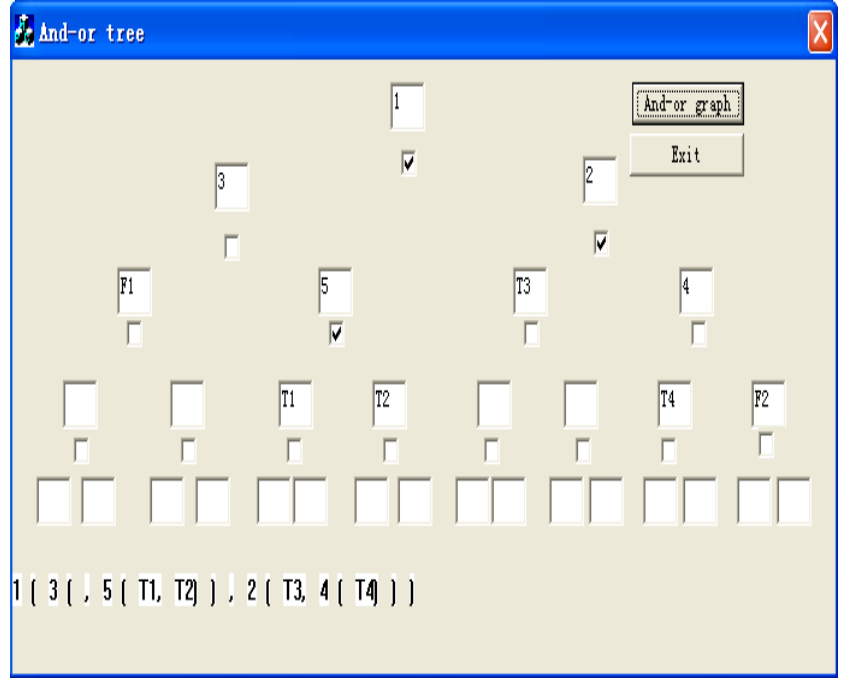

Fig.1 and-or graph search interface

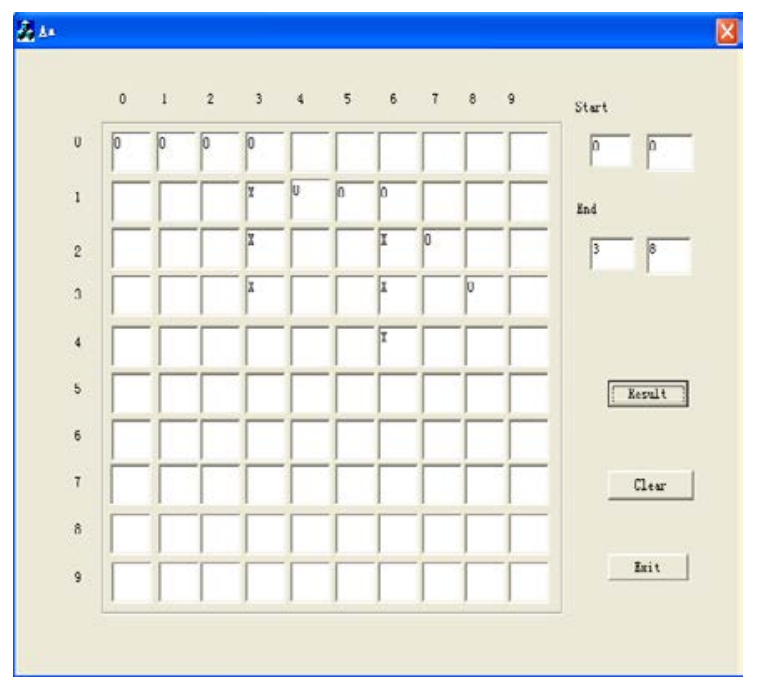

Fig.2 A* algorithm search interface

\section{The application examples about artificial intelligence}

In the teaching process, we change complicated and abstract theory into visual data of the application examples, permeate the theory in practice, intersperse in the practice theory, pay attention to the combination of theory and practice. We cultivate students' engineering practice ability and make student that is willing learning really.

The choice of application examples. Application examples are divided into two types. One kind is the application of basic knowledge. One application correspond an intelligence algorithm. Another kind is the application of comprehensive knowledge. One application correspond many intelligence algorithms. The principle of selecting application examples is that the students are familiar with the steps that are similar with an algorithm. It is the first kind that a most important application is selected by us. These application examples can improve the students' interest.

Teaching content and application examples. Most intelligent algorithm has at least one or more application examples. For example, the examples of graph search is Maze, tree state graph search is Eight Digit, game tree search is tic-tac-toe, minimum cost search is minimum short route, and-or graph search is Hanoi Tower, genetic algorithm is count maximum, machine reasoning based on generative is Animal identification expert system, Artificial Neural Network is digital identify, etc.. These application examples are in the demonstration system. Fig.3 shows a last result interface for tic-tac-toe. Fig. 4 shows a reasoning result interface for animal identification.

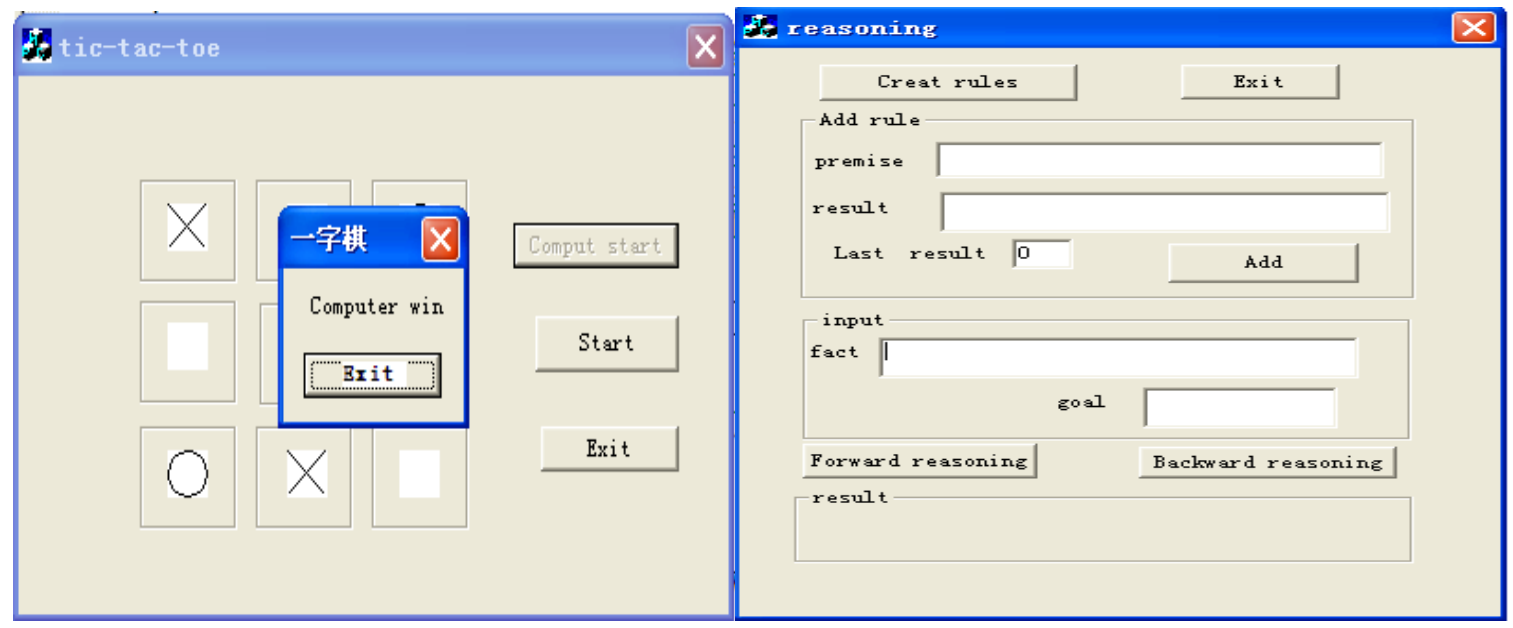

Fig.3 tic-tac-toe interface

Fig.4 animal identification interface 


\section{The artificial intelligence training system}

We designed a training system for student. The system is based B/S using VS2010 C\#. Students can review the algorithm of the artificial intelligence after school. This system can automatically judge the students' results. The students can also modify the training topics. Fig.5 shows a tree search training interface. The upper left part of the interface shows the training algorithm. The upper right part of the interface shows the steps of selecting algorithm.

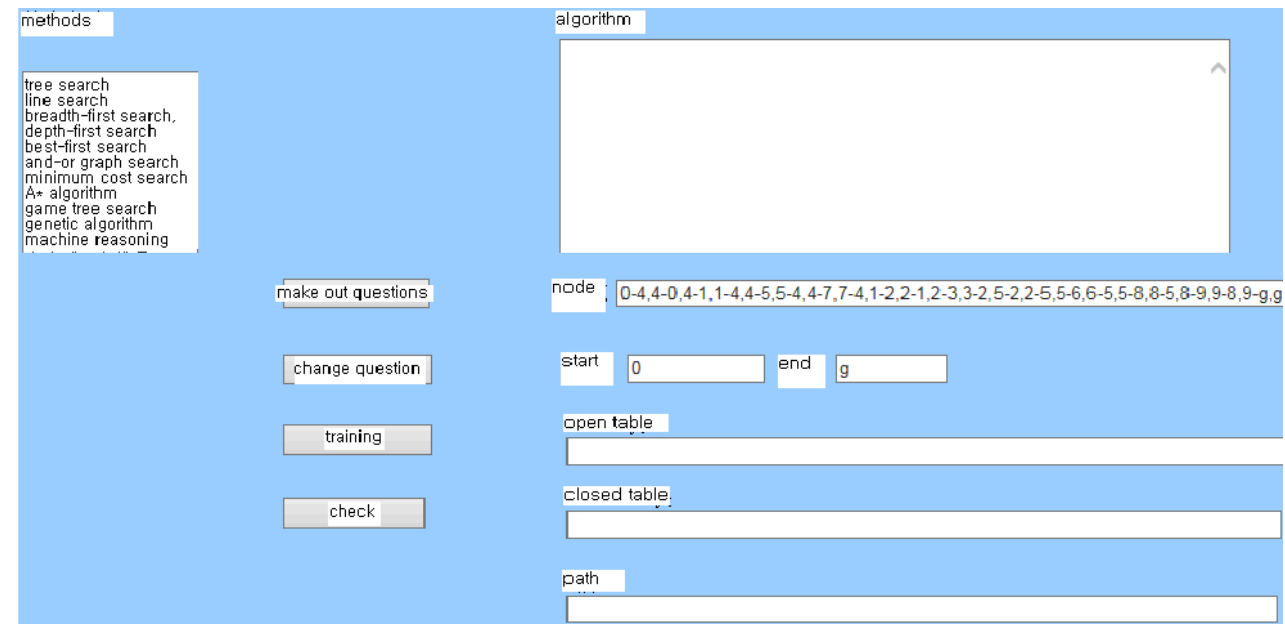

Fig.5 tree search training interface

\section{Summary}

With the development of information technology, the artificial intelligence technology is also developing, and is increasingly used in various fields. Through the process of many years teaching experience of artificial intelligence, we designed a variety of teaching software. The software has been used nearly five years in the practice teaching of the school. We try to stimulate students' interest in learning and improve students' learning ability, practical ability and innovation ability and lay a solid foundation for training high-quality talents.

\section{Acknowledgements}

This work was financially supported by the Department of Education of Jiangxi Province of China (JXJG-12-8-1).

\section{References}

[1] D.D. Yang, H.X. Sun, P. Yang and L. Zhang, submitted to Computer Education (2011)

[2] T. Liu, and Y.W. Liu, submitted to Journal of Computer Education (2012)

[3] R. Xie, and X. Li, submitted to Journal of Computer Education (2014)

[4] J.B. Ma, Y.L.Li and Y. Gao, submitted to Journal of Agricultural University of Hebe(2011)

[5] M.H.Wang, submitted to Journal of Heilongjiang College of Education(2013)

[6] J.H.Duan, S.S.Zhang, J.G. Wei and Q.Mao, submitted to Journal of Computer Education (2013)

[7] B. Chen, submitted to Computer Engineering \& science (2014) 